\title{
Miradas a la investigación educativa desde el género: un estado de conocimiento
}

\author{
María Silvia Aguirre Lares \\ Romelia Hinojosa LUJÁN \\ Profesoras investigadoras \\ Centro de Investigación y Docencia y \\ Centro Chihuahuense de Estudios de Posgrado
}

\section{Resumen}

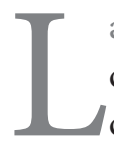

a elaboración de un Estado de Cono-

cimiento de la investigación educativa,

con una mirada de género, en el es-

tado de Chihuahua, es el objetivo central de esta investigación. Mediante la valoración de los logros, tendencias temáticas y metodológicas, los avances, carencias y retos, permitió establecer claramente la agenda pendiente en torno a la investigación sobre educación y género. A través del análisis hermenéutico de las producciones de la entidad surgen tres subcampos en la investigación de los géneros: el histórico, el de las identidades y el de las prácticas educativas.

Palabras clave: género, investigación educativa, historia de la educación, identidad, prácticas educativas.
La mirada del género es un tema emergente en la institucionalización de las políticas educativas. Cada vez más la tendencia en todos los niveles de la misma, es su enunciamiento, legislación, programación y operativización (Fuentes Molinar, 1984).

Dentro de este entramado de discursos, proyecciones y ejecuciones de la política educativa, se destaca un aspecto nodal para la toma de decisiones: la investigación. El Estado mexicano reconoce la necesidad de investigar la temática de género y lo expresa abiertamente en las demandas sectoriales establecidas por CONACYT/SEP en el 2007. Establece como productos de investigación esperados, los diagnósticos y estudios de factores asociados a la atención, equidad y calidad educativos, así como análisis de situaciones concretas de diversidad y desigualdad social y su impacto en

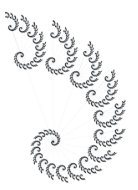


las condiciones de aprendizaje del alumnado (CONACYT/SEP, 2007).

El reconocimiento de una sociedad diversa y multicultural atañe no solamente a aspectos de raza, clase social, lenguaje, cultura, preferencia sexual, discapacidad o género. Aunque el término sea más usualmente utilizado en torno a las diferencias culturales o de otro tipo de diversidad (Kincheloe y Steinber, 1999).

Derivada de decisiones consensadas en la aldea global, se posiciona en nuestro país como un tema recurrente en el quehacer educativo la diversidad acotada en expresiones de género. La posición del Estado Mexicano es cada vez más congruente en torno al reconocimiento de la diversidad como riqueza social; y dentro de ella podemos ubicar a la diferencia que culturalmente se le asigna a los comportamientos, roles y relaciones jerárquicas, por el hecho de pertenecer a uno u otro sexo.

Dentro de este contexto complejo y fértil para la realización de una investigación que oriente la toma de decisiones de las autoridades educativas y los académicos interesados en la temática, se inserta el presente artículo que se desprende de la elaboración de un estado de conocimiento de la investigación educativa bajo la mirada del género, en el estado de Chihuahua.

Es necesario aclarar que la elaboración de los estados del conocimiento de la investigación educativa en el estado de Chihuahua fue un trabajo interinstitucional que aglutinó a más de 30 investigadores y docentes de nivel superior. La idea fue trabajar las áreas propuestas de forma organizativa por el Consejo
Mexicano de Investigación Educativa (COMIE). El presente artículo recopila la experiencia del equipo que trabajó el campo Educación y género.

Los antecedentes en la elaboración de estados de conocimiento se remontan hacia 1981, cuando en el I Congreso Nacional de Investigación Educativa (CNIE) se realizaron algunos documentos breves que se constituyeron en los prolegómenos de esta tradición que actualmente está consolidada.

En 1993 se realiza el II CNIE y nuevamente se elaboran documentos base para la discusión que fueron llamados formalmente estados del conocimiento y cristalizaron en la publicación de 29 cuadernos de trabajo. Posteriormente, en el año 2000 se iniciaron los trabajos para valorar la producción investigativa del periodo comprendido entre 1992 y 2002. En el VII CNIE se concretó la publicación de 14 libros sobre los estados de conocimiento (Martínez, 2009).

A nivel nacional, en diversas entidades federativas, se han desplegado esfuerzos de investigadores locales por realizar estados del conocimiento con perspectivas estatales. Uno de ellos fue el realizado en el marco de la elaboración de los estados de conocimiento a nivel nacional para la década de los noventa. Se anotaron quince entidades. Al final el trabajo estuvo constituido por 13 de los 31 estados del país, de los cuales sólo ocho entregaron informe para publicación (Martínez, 2009). Chihuahua no participó en esa ocasión en la integración y construcción del estado de conocimiento nacional. 
El campo de género inició su presencia en los estados del conocimiento elaborados por COMIE. La colección "La investigación educativa en los ochenta. Perspectiva para los noventa" incorpora en el tomo Educación, cultura y procesos sociales los estudios relativos a la educación, género y mujer. Una década después, en el 2003 emerge el campo Educación y género. Sin embargo, observamos con extrañeza que en la reestructuración de las áreas que se realizó en Pachuca en 2006, el campo género se diluyó de forma transversal en las demás áreas. Esta última circunstancia motivó una fuerte discusión en Chihuahua, ya que en esa misma época iniciaron los trabajos estatales para elaborar el estado de conocimiento de la investigación educativa en la entidad. De inicio la decisión estatal fue acogerse a la organización de los campos y las áreas de COMIE, entonces el dilema para el equipo responsable del campo de género era transversalizar los productos localizados en este tema en todos los campos, incorporarse a un área afín, o bien, abrir un nicho propio. El acuerdo que se logra, con apoyo del equipo estatal de investigación, es:

Abrir explícitamente un campo que recogiera la producción estatal sobre Educación y género (...) porque permite dar cuenta de un trabajo sostenido de diversas personas que han aportado a la producción estatal en el campo, además, nos parece que la transversalización en estos momentos, provocaría la invisibilización de un tema que, además de aportar un sólido corpus teórico para estudiar analíticamente la diferencia social, brinda la posibilidad de analizar el género como categoría social (Aguirre, et.al, 2007: 26).

Encontramos como único antecedente de elaboración de un estado de conocimiento estatal de la temática de género al trabajo elaborado en el estado de Nuevo León (Lamadrid, 2001). En él se analizan las experiencias y la formación de las personas participantes en el campo, extrapolándolo a ámbitos no necesariamente educativos. Es decir, no analiza en forma precisa la investigación educativa de género, sino todo el campo de género en lo general. Es esta la gran diferencia entre el trabajo realizado por personal regiomontano y el nuestro.

Las preguntas que guiaron la investigación fueron: ¿cuántas investigaciones sobre género en la educación se han realizado a nivel estatal? ¿cuáles son las que se han producido? ¿a qué niveles y contextos corresponden? ¿bajo qué líneas metodológicas y enfoques teóricoconceptuales se han adscrito? ¿qué otro tipo de producciones académicas se han hecho?

La idea no era pues, hacer un listado de las investigaciones que utilizaran conceptualmente la teoría de género realizadas en el ámbito educativo. Nuestro planteamiento fue interpretar la producción en torno al campo, desentrañar las recurrencias y divergencias, analizar el detalle, sacar a la luz lo no evidente. Por estas consideraciones se puede ir perfilando el paradigma epistemológico que fundamentó la misma: el interpretativo. 


\section{El posicionamiento teórico metodológico}

Es imposible realizar un trabajo de esta naturaleza sin que influyan los elementos epistemológicos, teóricos, históricos, políticos e ideológicos de las investigadoras. Bajo esta premisa se abandona por completo la posibilidad de lograr un conocimiento "objetivo".

Al hablar de interpretación de investigaciones, la elección obligada y natural es el método hermenéutico. "La hermenéutica se define como la teoría y práctica de la interpretación" (Álvarez-Gayou, 2003: 80). Optamos entre las diversas hermenéuticas por la analógica porque:

La hermenéutica analógica intenta sortear las extrapolaciones tan generalizadas en las temáticas sobre género, y nos proporciona una visión más integradora de la realidad de este tema ya que, tal constructo, que funge como modelo teórico de interpretación, muestra su preocupación y su central intención que es la comprensión de las realidades. En la analogía se reúnen las propuestas univocistas y las equivocistas, las que pretendes ser universales y las que apuestan por ser específicamente particulares y absolutamente diferentes (García y Guerrero, 2005: 6).

Como no se busca "la verdadera interpretación", pero tampoco negar todo tipo de referencia, la hermenéutica analógica es un piso conceptual que proporcionó criterios de inclusión y de exclusión en el campo. En los de inclusión se consideró el planteamiento del problema y el posicionamiento teórico de las autoras y los autores, principalmente, también que el objeto de estudio considerara a los sujetos sexuados y las representaciones de género que de él se derivaran. Los límites de la exclusión fueron la ausencia de una perspectiva de género o el estudio no diferenciado de los hombres y las mujeres.

Conceptualmente consideramos que el corpus teórico del género se refiere al conjunto de categorías que abordan analíticamente la diferencia sexual (Barquet, 2002), para dar cuenta de la subordinación y dominación que existe en las relaciones de poder que hombres y mujeres ponen en práctica en los ámbitos público y privado. El género está referido a las formas históricas y sociales en que las personas construyen sus identidades, interactúan y organizan su participación en la sociedad. Al ser una categoría configurada por el tiempo y el espacio, ésta se reproduce y se transforma continuamente. El género entonces, no puede ser considerado estático o "natural", como aseguran los que asignan estereotipos y roles separados a los hombres y las mujeres.

La categoría género es un aporte revolucionario del feminismo, porque posibilita una visión crítica. Esta visión es conocida como perspectiva de género, Marcela Lagarde nos dice que esta herramienta política y conceptual:

Permite analizar y comprender las características que definen a las mujeres y a los hombres de manera específica, así como sus semejanzas y diferencias (...) 
Analiza las posibilidades vitales de las mujeres y los hombres: el sentido de sus vidas, sus expectativas y oportunidades, las complejas y diversas relaciones sociales que se dan entre ambos géneros, así como los conflictos institucionales y cotidianos que deben enfrentar y las maneras en que lo hacen (Lagarde citada en Bustos, 2003: 5)

Este posicionamiento nos permitió asumir una actitud comprensiva hacia las perspectivas de las personas que contribuyeron con sus textos a la configuración del campo. Todos los aportes son considerados valiosos, tanto las investigaciones realizadas por estudiantes de maestrías y doctorados, como las realizadas por investigadoras de reconocida trayectoria estatal y nacional. En un campo temático que emerge de la denuncia y la lucha de las mujeres por ser consideradas protagonistas sociales, lo menos que se puede hacer es registrar la voz de quienes buscan los significados de sus sentires y sus acciones.

Para facilitar el procesamiento de los textos en análisis, utilizamos tres tipos de ficha que adecuamos de los propuestos por el Consejo Mexicano de Investigación Educativa (COMIE) en otras experiencias semejantes: la bibliográfica, de clasificación y de análisis de contenido. Dichas fichas permitían focalizar elementos centrales del análisis de las investigaciones y fueron adecuadas a las características del campo.

Las delimitaciones contextuales que establecimos, giraron en torno a lo que conceptualizamos como investigación. Definimos a la investigación educativa como un ejercicio de búsqueda sistemática e intencionada de respuesta a interrogantes planteadas en relación al quehacer educativo, que no se quede en una respuesta sobre la apariencia del fenómeno. El tipo de respuestas encontradas podrían darse a partir de descripciones, explicaciones, o de acciones específicas que modifiquen el hecho educativo. Los trabajos podrían estar publicados o no y el formato de presentación podría ir, desde ensayos argumentativos y ponencias, hasta reportes de investigación. Tomamos esta decisión, a la luz de valorar este primer estado de conocimiento como la fase inicial de un proceso continuo, en el que en estados posteriores se podrán introducir criterios más rigurosos para la inclusión de trabajos.

De la misma forma decidimos no realizar un corte temporal en torno a la producción investigativa de nuestra entidad, puesto que no es muy nutrida y no se había realizado ninguna experiencia de este tipo. El corte geográfico lo ofreció el estado de Chihuahua, particularmente en los municipios con presencia de instituciones de nivel superior que tienen como función sustantiva la investigación.

\section{El comportamiento del campo}

Para realizar la presente valoración de la producción investigativa y de difusión en el estado de Chihuahua, cuya temática diera respuesta a las preguntas de nuestro estudio, transitamos primero por la búsqueda y localización de documentos. En el primer recorte localizamos 81 escritos, de los cuales fueron excluidos 25 por varias razones: algunos de 
ellos no se referían al hecho educativo, otros no acudían a la perspectiva de género para el estudio de la realidad, no arrojaban resultados relacionados con la problemática de las relaciones de género o bien, no documentaban las identidades y prácticas de los hombres y las mujeres.

Por otra parte, algunos artículos de revistas reflexionaban en torno a la temática de género con grandes discusiones teóricas; sin embargo fueron producidos por personas de otras entidades o países. En otros casos no se enfocaban hacia el análisis del proceso educativo por lo que también se decidió excluirlos.

Finalmente la producción analizada quedó integrada como lo muestra la gráfica 1 .
Se puede apreciar que la mayor parte de la investigación se realiza con la intención de obtener algún grado académico, además de ser escasa puesto que estamos hablando de más de una década de producción. La funcionalidad que se le atribuye no es la de informar a los actores educativos, tomadores de decisiones o al público en general de los problemas o hallazgos obtenidos en el proceso heurístico.

Respecto al período de productividad, el primer trabajo localizado corresponde a 1994, por lo que este estado de conocimiento cubre 14 años. Se observa una mayor productividad en el 2003, seguido del 2004 y 2006. De cualquier manera, la mayor parte se concentra en los últimos cinco años.

\section{GRÁFICA No. 1}

\section{PRODUCCIÓN ANALIZADA}

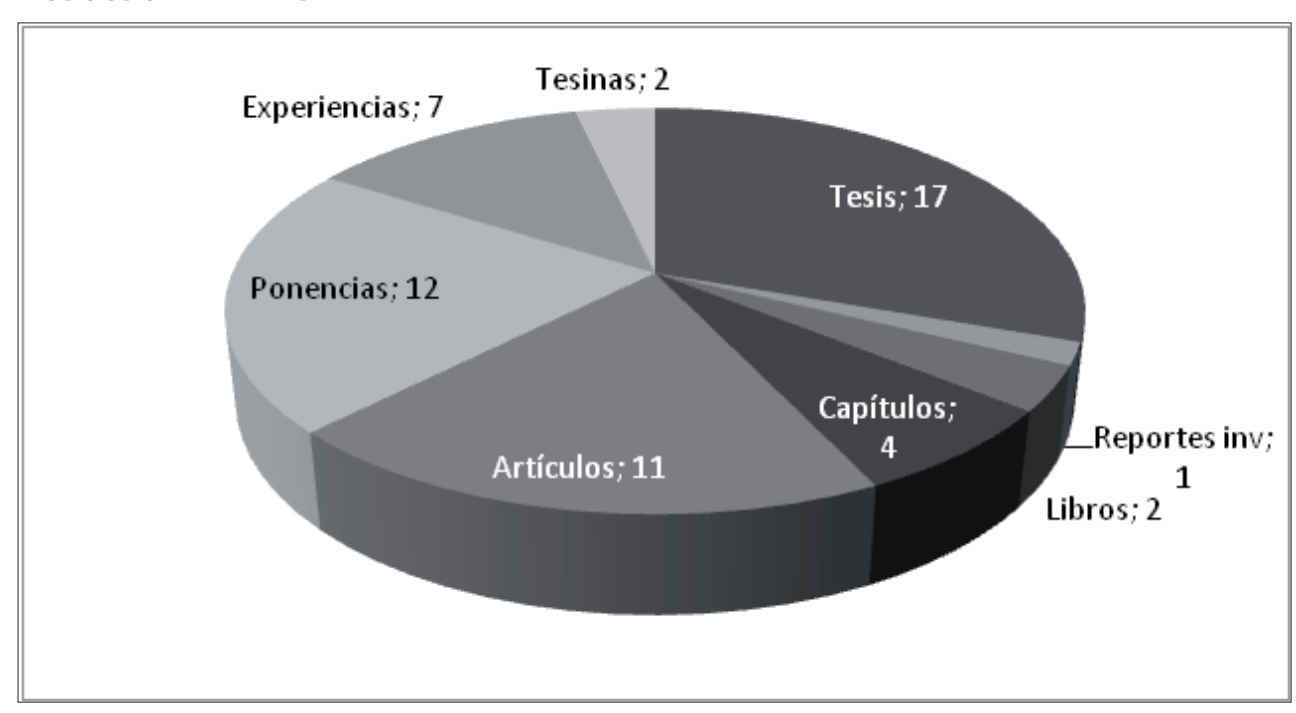


GRÁFICA No. 2

\section{AÑOS DE PUBLICACIÓN}

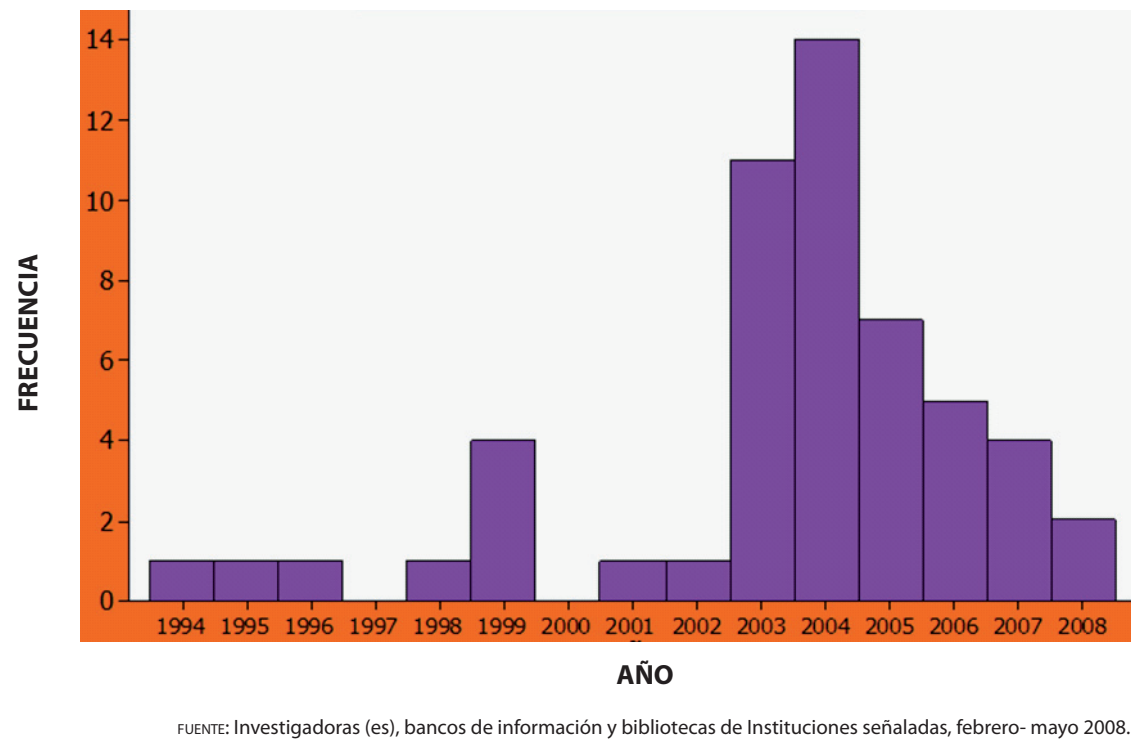

El comportamiento del período se muestra a detalle en la gráfica No. 2:

De un total de 56 trabajos, los años más prolíficos son 2004, 2003 y 2005 con 14, 11 y siete referencias respectivamente. Durante el período de 2003 a 2005, se organizan varios eventos académicos que proporcionan 12 ponencias y siete relatos de experiencias publicadas lo que aumentó considerablemente el bagaje de este campo. Le sigue el 2006 que se destacó por la publicación de libros con la temática de mujeres. En el 2007 se destacan cinco tesis y una tesina del campo, por lo que se observa una tendencia a la alza de producción dentro del mismo, sin embargo en el año 2008, hasta el mes de mayo, únicamente se habían producido dos tesis. De este conteo queda fuera el informe de investigación (documento multicopiado), porque no fue posible determinar su fecha de publicación.

Otro factor analizado corresponde al sexo de las autoras o autores de las producciones. Llama la atención que las investigaciones fueron elaboradas, en todos los casos por mujeres; los artículos de revistas son elaboradas en un $88 \%$ por mujeres y el $12 \%$ por hombres. Con respecto a las memorias y relatos de experiencias, los porcentajes no son muy diferentes, el $70 \%$ son trabajos de mujeres, el $20 \%$ de hombres y un $10 \%$ de autoría colectiva mixta. En torno a las publicaciones en periódicos la tendencia es completamente contraria a la de las tesis, puesto que el $100 \%$ es de autoría varonil. Los relatos de experiencias, siete en total, 
representan el grupo con menor dispersión: $57 \%$ elaborados por maestros y $43 \%$ por mujeres. Sigue entonces considerándose como un campo eminentemente femenino.

Sobresale que toda la investigación analizada, 24 productos, es realizada por mujeres. Ellas recurren de manera predominante al método histórico, utilizado en once estudios; las autoras se apoyan en técnicas variadas como la historia de vida, historia oral, análisis de fuentes primarias y secundarias. En segundo lugar tenemos cinco investigaciones donde predomina el enfoque etnográfico; en estos casos la observación participante, las entrevistas y el grupo focal son las técnicas más utilizadas.

Los diseños de corte cuantitativo suman cuatro, de ellos tres son a nivel descriptivo y uno a nivel correlacional; en estos casos las técnicas más frecuentes son las encuestas, escalas, cuestionarios, y test. Sólo una investigación es de corte emergente, esto es, recurre tanto al análisis de corte estadístico como al interpretativo. A la investigación-acción recurre una sola de las tesistas, con este diseño pretendía transformar su práctica docente, por lo que podemos pensar que es una investigación aplicada. Finalmente, encontramos dos investigaciones con inconsistencia en el diseño porque dicen acogerse al enfoque cualitativo, pero al análisis de la información se le da, predominantemente, un tratamiento estadístico.

Observamos pues que la mayoría de la investigación educativa, organizada en el campo de Educación y género, es realizada bajo enfo- ques cualitativos, se dirigen hacia estudios de casos y los objetos de estudio se refieren a problemáticas de las mujeres. Estas indagaciones dan cuenta de diversas formas de cuestionamiento que las investigadoras hacen sobre la realidad, con el ánimo de reconocer la cotidianidad y las formas de cómo se manifiestan las relaciones de género en las diferentes facetas de la vida de las personas.

El peso mayor lo constituyen las tesis elaboradas con el propósito de obtener el grado de maestría; y en mínima proporción de doctorado. Los estudios son realizados de manera individual, en ningún caso se localizaron experiencias interinstitucionales o de equipos de investigación. Puede deducirse que el financiamiento de la investigación es asumido por las propias tesistas ya que solo dos personas recibieron patrocinio institucional. Desde nuestra óptica no existe una comunidad investigadora consolidada en nuestra entidad. En la mayoría de los casos son investigaciones únicas, incluso podemos adelantar que esta circunstancia seguirá mientras la investigación sea vista con fines de titulación. Otra conclusión a la que arribamos es el escaso reconocimiento que las autoras del campo tienen entre ellas. No acuden a estudios estatales como antecedentes de los propios, por lo tanto existe el riesgo de estar abordando la problemática educativa desde ángulos ya agotados, limitando la emergencia de nuevas categorías.

En lo referente a la difusión de la investigación, encontramos que son pocos los esfuerzos para la divulgación de los resultados. 
La mayoría de las instituciones cuentan con el formato de revista, donde pueden publicar los miembros de las instituciones o colaboradores externos; sin embargo, no abundan los reportes o escritos que retomen como propósito las experiencias en investigación, a excepción de la UACJ que parece tener muy consolidada su política editorial.

Constituyen esfuerzos importantes los libros editados a partir de investigaciones; en este caso se localizaron dos tesis (Martínez, 2006 y Estrada, 2004); también están cuatro capítulos de libro que brindan información para nuestro campo: dos aportados por una investigadora estatal (Franco, 2001 y 2006) y dos más destinados a su divulgación nacional (Calvo, 2003 y Arredondo, 2003). Sin embargo, los pocos ejemplos de divulgación provienen de los intentos que las personas hacen para que su trabajo se conozca, sintiéndose la necesidad de mayor apoyo institucional.

El estado de conocimiento de la investigación educativa en el campo Educación y género, se organiza en cuatro grandes apartados o subcampos. Éstos, fueron elaborados individualmente por las integrantes del equipo responsable del campo, aunque bajo una estrategia de análisis acordada en el colectivo. Argelia Ávila Reyes realizó la descripción estadística del campo; Romelia Hinojosa Luján organizó el apartado de historia de las mujeres; María Silvia Aguirre Lares trató el tema de las identidades de las y los sujetos de la educación y María Cristina Chávez Rocha expone las prácticas educativas. Una breve descripción de estos apartados se realiza a continuación.
La historia de las mujeres es el apartado más consolidado, ya sea porque es ahí donde se encuentra el mayor número de publicaciones, como por su cuidadoso manejo metodológico y porque la mayoría son realizadas por investigadoras e investigadores de reconocida trayectoria académica o por tesistas que lograron estudios a profundidad y novedosos en el área.

No se alude concretamente a alguna categoría en lo particular, sin embargo los fenómenos que se describen y analizan son: patriarcado, reproducción de roles de género, ámbito privado, feminización de la enseñanza, inserción de la mujer en el ámbito público y rompimiento de estereotipos.

Otro tema recurrente es el que indaga en las identidades de las y los sujetos de la educación. Las categorías que surgen del análisis se refieren a la feminización de la enseñanza y de las carreras en educación superior; ahí la mirada está puesta en el imaginario social que relaciona el ejercicio de la docencia y la elección de carreras profesionales, con los "atributos naturales de las mujeres", convirtiéndose estos estereotipos en limitaciones para la propia población femenina. Otra categoría muy consolidada se encuentra en las investigaciones que estudian las relaciones de poder y las estrategias a las que recurren las mujeres para resolver los obstáculos que enfrentan en su ascenso laboral. Sobresale la denuncia que las autoras hacen de la categoría techo de cristal que limita el crecimiento de las mujeres. Cierra este apartado el análisis que las investigadoras del campo realizan sobre la figura de la 
madre, sus vicisitudes para sortear las demandas familiares y sociales sobre el desempeño de sus roles tradicionales de madre, esposa y ama de casa, al mismo tiempo que las necesidades económicas y las aspiraciones personales le llevan a desempeñarse también como trabajadora asalariada. Aquí las investigadoras, nos presentan la persistencia de la doble y triple jornada que coloca a las madres de familia en una situación de culpa permanente.

En el tema de las identidades de las y los sujetos de la educación, está escasamente tratada la masculinidad y los estudios de hombres sobre los hombres. Los pocos trabajos encontrados - una tesis y tres ponencias- indagan sobre el desempeño de los docentes y los retos que la masculinidad hegemónica enfrenta en la actualidad.

El tercer tema encontrado en las investigaciones se refiere a las prácticas educativas. En él se centra la mirada en los actos de reproducción de la institución, lo que nos permite identificar la persistencia de una práctica sexista que reafirma los roles y estereotipos tradicionales de género. Falta documentar las acciones de resistencia del alumnado, las transformaciones que está teniendo la cultura en torno a las relaciones de género o aquellas experiencias coeducativas que van en pos de una equidad entre los géneros.

La interpretación del campo nos permite dilucidar una perspectiva teórica y conceptual que cobija las investigaciones, artículos y ponencias analizadas. Si bien se supera la visión esencialista biológica y se explica el gé- nero a partir de la construcción social e histórica, todavía persisten las ideas de que los estereotipos y roles de género son inalterables, lo que impide mirar los múltiples procesos y estrategias a través de los cuales los géneros se transforman y se trastocan, de igual manera los estudios no se dirigen a localizar la diversidad, se atiende a una perspectiva homogeneizadora que normaliza a los hombres y las mujeres, explicación que pudiera derivarse de una visión sociológica reproduccionista o bien, de un esencialismo social. Existen trabajos sobresalientes por el cuidadoso proceso de análisis, que se apoyan en diferentes áreas del conocimiento social, además del fuerte apoyo que se toma de la perspectiva de género, en estos casos se logran niveles interpretativos que nos ayudan a comprender mejor la problemática estudiada. Pero también encontramos investigaciones que utilizan la categoría de género de manera intuitiva, sin profundizar teóricamente en torno de la misma, sin aprehenderla a la hora de realizar el análisis.

En términos generales, nos parece que predomina el ideal de la igualdad, aunque en algunos trabajos de investigación, todavía se presenta confusión en los conceptos de desigualdad o diferencia, lo que nos indica que el debate político y el ontológico no está resuelto. Existe además poco reconocimiento del feminismo como productor de la perspectiva de género y, en la mayoría de las investigaciones, hace falta anclar la perspectiva de género a un piso teórico más sólido que puede ser proporcionado por la sociología, la historia o la psicología, entre otras ciencias sociales. 
Algo que llama la atención es que se estudian las diferentes problemáticas a partir de conceptos o categorías ya construidas y "legitimadas" en los estudios de género; en este sentido permanecemos en la periferia del conocimiento, ya que los grupos consolidados de investigación parecieran estar en el centro de la República, de donde proceden la mayor parte de las publicaciones nacionales en el tema. Coincidiendo con esta idea, en nuestra entidad reproducimos el fenómeno del centralismo ya que ciudad Juárez y Chihuahua concentran la mayor parte de la producción quizá porque tienen la mayor cantidad de población en el estado y cuentan además con la mayor oferta educativa.

\section{La agenda del campo}

En 1995 México signa la declaración de Beijing donde se compromete a establecer acciones para evitar la discriminación y la desigualdad en el acceso y permanencia de las mujeres en la educación. Uno de los compromisos o medidas, entre muchas más, que los Gobiernos, autoridades educativas y otras instituciones educativas y académicas deben de adoptar, dice a la letra:

g) Apoyar y realizar estudios e investigaciones sobre el género en todos los niveles de enseñanza, especialmente en el nivel de postgrado de las instituciones académicas, y aplicarlos a la elaboración de los currículos, incluidos los de estudios universitarios, en libros de texto y material didáctico y en la formación de personal docente.
Han pasado quince años desde entonces $y$ aunque sabemos que el nivel discursivo de la política educativa pocas veces tiene que ver con la operación de programas y acciones específicas que permitan resolver las grandes problemáticas educativas, nuestros sueños se renuevan a partir de la realización de este estudio. Consideramos que la investigación educativa puede proporcionar derroteros por los que las políticas educativas habrán de transitar, siempre y cuando se atienda a lo que de ella emerge.

La valoración del campo permite formular algunas conclusiones, así como esbozar áreas de intervención tanto para las instituciones educativas que realizan investigación, como para los responsables de la política educativa en el estado, y para los y las investigadoras.

Como ya se mencionó, las investigaciones encontradas recurren a metodologías cualitativas. Es decir, recurren a la interpretación simbólica de las interacciones culturales desde la perspectiva de género. Este hecho puede significar ventajas: conceptualiza la realidad desde un plano complejo; asume la subjetividad del conocimiento producido; profundiza en las interacciones e interpretación de los hechos sociales. Sin embargo, tiene sus limitantes: no se pueden abarcar y describir grandes grupos poblacionales; con una gran limitante en las generalizaciones que se obtienen de este tipo de investigación. Por otro lado, se llega a la denuncia, a la descripción pormenorizada de los hechos educativos -sexistas por ejemplo-, pero no se hace más nada ante ellos, se asume una actitud descriptiva-contemplativa. 
Por estas consideraciones es que el abanico metodológico se puede ampliar y debemos transitar en varios caminos: primero, realizar estudios cuantitativos que permitan dimensionar grandes grupos poblacionales, hacer generalizaciones sobre el estado de los fenómenos objeto de estudio, sobre violencia de género en las escuelas, identidades de género en los y las jóvenes, desarrollo moral del profesorado focalizado en la aceptación a la diversidad. Segundo, asumir metodologías que impliquen la transformación del hecho que se estudia, no únicamente su interpretación y denuncia. Es decir, promover mayor número de trabajos de investigación bajo un paradigma crítico y transformador, que establezca la necesidad de hacer replanteamientos y reconstrucciones de las relaciones sociales entre los géneros en el hecho educativo a través de la investigación participativa o investigaciónacción, por ejemplo. Tercero, partir de que la realidad no es única y para su comprensión amplia se puede transitar hacia opciones metodológicas o técnicas de complementariedad.

La investigación que no se difunde, no existe. Ante este planteamiento es necesario fortalecer los programas de divulgación de las instituciones de nivel superior. Es increíble que las producciones de tesis no sean objeto de ensayos o artículos en las revistas de las escuelas. Más allá de esto, son pocas las iniciativas para difundir resultados de investigación, sólo encontramos dos eventos estatales convocados ex profeso sobre la temática y pocas participaciones en foros y otros eventos nacionales. Definitivamente se debe crear un proyecto que vaya más allá del plano institucional, y que recurra a instancias gubernamentales en el que su tarea sea la difusión de los hallazgos en el ámbito educativo estatal.

Ese mismo proyecto podría abarcar otros aspectos en los que se encontraron fallas o deficiencias. La comunicación y el trabajo interinstitucional es una de las principales debilidades del campo. La ausencia de trabajos con estas características es una muestra de ello. Entonces habría que tender puentes y nexos de comunicación entre las instituciones de nivel superior en los que se hable de compromisos de trabajo, multidisciplinariedad y trabajo transinstitucional. La diversidad enriquece, la cooperación engrandece ¿por qué no practicarla? La presente investigación, es una muestra de que se puede realizar un trabajo interinstitucional, conviene entonces promover este tipo de prácticas, pensando en un futuro conformar una red de investigación sobre estudios de género en educación.

\section{NOTAS}

1. Se distingue entre estado de conocimiento, estado de la investigación y estado del arte. El estado del arte se dirige hacia contextos teóricos mucho más amplios (internacionales y nacionales) en los que se recuperan todos los saberes publicados y divulgados en torno a cierta temática; el estado de la investigación se dirige hacia las condiciones de producción de la investigación; por último un estado de conocimiento lo asumimos como una valoración sistemática de la producción intelectual sobre un campo de investigación durante un tiempo cronológico determinado. El estado del conocimiento tiene la intención de detectar las líneas de estudio trabajadas y las ausentes, los referentes teórico-metodológico privilegiados, la tipología de la producción que se ha generado, todo ello con la finalidad de generar un conocimiento sistemático, analítico, crítico y propositivo en torno a la producción investigativa de un determinado campo. Aguirre, S. Ávila, A. Hinojosa, R. (2007). Aportes para el debate sobre el estado de conocimiento en el campo Educación y género, (Chihuahua, Méx.), Acoyauh, núm. 39, pp. 26-35 


\section{Bibliografía}

Álvarez-Gayou Jurgenson J. L. (2003) Cómo hacer investigación cualitativa. Fundamentos y metodología, México, Paidós Educador.

Arredondo, A. (2003). "Un atisbo a una escuela de niñas al comienzo del siglo XIX”. En Arredondo A. (ed.), Obedecer servir y resistir. La Educación de las mujeres en la historia de México. México: UPN/Miguel Ángel Porrúa Grupo Editorial (pp. 99-116)

Barquet, M. (2002). Reflexiones sobre teoría de género hoy (La Paz, Bolivia), en Umbrales, núm. 11, CIDES-Universidad Mayor de San Andrés

Bustos, O. (2003). "Género y socialización: familia, escuela y medios de comunicación”. En SEP, Construyendo la identidad de género en la escuela primaria. Material de la o el participante. México, Secretaría de Educación Pública e Instituto nacional de las Mujeres, pp. 2-27

Calvo, B. (2003). "Marina y sus techos de cristal. Las vicisitudes de una maestra". En Cortina R. (comp.) Líderes y construcción de poder. Las maestras y el SNTE, (pp. 67-87). México, D.F. Editorial Santillana.

Estrada, S. (2004). Con hilos de colores. Cinco mujeres maestras en el municipio de Guerrero. Chihuahua, México: Doble Hélice.

Franco, M. (2001). El trabajo de las mujeres, actividad poco valorada. En Franco M. (ed.), San Diego de Alcalá. Un Pueblo que se resiste a morir. Chihuahua, México: Doble Hélice (pp. 71-83).

(2006). Formación de Educadoras. En Franco M. (ed.), Imágenes, voces y recuerdos. Una historia de la Escuela Normal del Estado de Chihuahua. Chihuahua, México: Doble Hélice (pp. 107-118).

Fuentes Molinar O. (1984) "La construcción, los niveles y los agentes de la política educativa, Notas para discusión" en Antología de Política Educativa, Maestría en Desarrollo Educativo, Centro Chihuahuense de Estudios de Posgrado, documento electrónico.

CONACYT/SEP (2007) Fondo Sectorial de Investigación para la Educación. Demandas específicas para el sector.http://www.conacyt.mx/Fondos/Sectoriales/SEP/ SEP-SEByN/2007-01/SEP-SEByN_Demandas-Especificas_2007-01.pdf consultada el 18 de noviembre de 2008.

García. D. E. y Guerrero A. L. (2005) Hermenéutica analógica y género. México, Editorial Torres Asociados.

Kincheloe J. y Steinberg S. (1999) Repensar el multiculturalismo, Barcelona España, Ed. Octaedro.

Lamadrid M. M. et. al. (2001) Informe del diagnóstico sobre el campo de los estudios de género en Nuevo León. Gobierno del Estado de Nuevo León/UPN. México.

Martínez R. (2009) Mínimos teóricos en la construcción de estados de conocimiento. Documento de divulgación de la Red Mexicana de Investigadores de la Investigación Educativa.

Martínez, S. (2006). Educación y Género. Docencia femenina en Hidalgo del Parral, Chih. (1631-1900). Chihuahua, México: Doble Hélice. 
